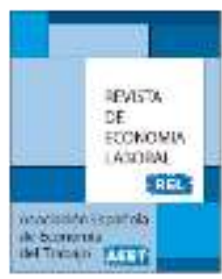

\title{
OFERTA LABORAL Y DEMANDA DE CUIDADOS INFANTILES EN LOS HOGARES ESPAÑOLES
}

\author{
Nuria Legazpe y María A. Davia \\ Universidad de Castilla - La Mancha
}

Recibido Diciembre 2017; Aceptado Enero 2018

\section{Resumen}

El objetivo del presente trabajo es analizar los patrones de participación laboral de madres y padres de niños pequeños y su vinculación con la demanda de cuidados formales e informales a lo largo del último cambio de ciclo en España. Para ello se explota la Encuesta de Condiciones de Vida (ECV) en el periodo 2005-2015. Una estimación simultánea del tiempo semanal que ambos padres dedican al trabajo remunerado y el que utilizan de cuidados formales e informales para sus hijos menores de tres años confirma la relación positiva entre la demanda de cuidados formales e informales y la participación laboral de madres y padres. Durante la crisis económica esta relación se hizo más débil en el caso de las madres y más significativa en el de los padres. Ello que podría apuntar a un cierto grado de implicación de los padres que perdieron el empleo durante la crisis en los cuidados de sus hijos pequeños, sustituyendo, esencialmente, a cuidadores informales.

Palabras claves: oferta laboral femenina, cuidados infantiles, división intra-hogar del trabajo.

Clasificación JEL: J13, J22.

(C) Revista de Economía Laboral 


\begin{abstract}
This paper aims to analyse labour market participation patterns in mothers and fathers of young children in Spain and how they are related to the demand for formal and informal child-care along the economic cycle. To this aim, we explore the Spanish Living Conditions Survey (2005-2015). We simultaneously estimate both parents' weekly hours in paid work and the time their under three year-old children spend in formal and informal child care. Our results confirm the positive relation between parental labour market participation and the demand for formal and informal care. During the economic crisis this relationship lost relevance in mothers while became more significant amongst fathers. The latter could point at a given degree of commitment in non-employed fathers with child care at home, substituting for non-parental (essentially, informal) child care.
\end{abstract}

Key words: female labour supply, child-care, intra-household division of labour JEL Classifications: J13, J22.

(C) Revista de Economía Laboral 


\section{Introducción}

El persistente avance de la participación laboral femenina y de la provisión de cuidados en España en las últimas décadas han transformado el mercado de trabajo español. Con la crisis económica se ha consolidado la presencia de las mujeres con hijos pequeños en el mercado laboral, que incluso se vio "espoleada" por el efecto trabajador añadido al inicio de la crisis de 2008. Esta transformación se ha logrado incluso a pesar de la pervivencia de un modelo familiar tradicional, marcado por la falta de flexibilidad horaria en el trabajo y una escasa implicación de los padres en los cuidados de los hijos, por cuanto muchas mujeres tratan de compatibilizar trabajo y familia recurriendo al apoyo de los abuelos.

El objetivo principal del presente trabajo es analizar los patrones de participación laboral de madres y padres de niños pequeños (menores de tres años) en España y su vinculación con la demanda que hacen de cuidados formales e informales para atenderles. Se espera comprobar que la presencia de niños pequeños en el hogar condiciona la oferta laboral de las madres en mayor medida que la de los padres. De igual modo, se buscan signos de un efecto trabajador añadido en las mujeres cuyas parejas no tienen empleo o posibles reducciones en las horas de trabajo en los varones en presencia de niños pequeños cuando sus parejas trabajan. Y, por último, se valorará si se ha producido un cambio en la relación entre la oferta laboral de padres y madres y la demanda de cuidados en situaciones de alto desempleo de los varones.

Para alcanzar estos objetivos se explotarán once olas de los ficheros transversales de la Encuesta de Condiciones de Vida (ECV) (2005-2015) que proporcionan información, para cada niño menor de 12 años, sobre el número de horas semanales en las que es cuidado fuera del hogar o las que pasa en centros escolares o de cuidados. Diferenciaremos entre cuidados formales (educación preescolar o infantil, centros de cuidado de niños fuera del horario escolar u otros centros de cuidado de niños) y cuidados informales (cuidadores profesionales remunerados en casa del niño o del cuidador (niñeras, asistentas, etc.) y otras personas que no obtienen remuneración (abuelos, otros parientes, amigos, vecinos, etc.)).

La estrategia analítica que se utilizará en este trabajo permitirá estimar la cantidad de horas a la semana que las madres y sus parejas dedican el trabajo remunerado y la de horas de cuidados formales e informales que demandan para cada uno de sus hijos menores de tres años. Todas ellas serán estimadas a partir de variables que describen 
cuatro ámbitos: características sociodemográficas y laborales, volumen y fuentes de ingresos, composición del hogar y características del entorno.

Este trabajo contribuye a la literatura sobre la interrelación entre la oferta laboral de las madres y la demanda de cuidados infantiles en España con la incorporación explícita en el análisis de la oferta laboral de los padres. Ésta se ha considerado tradicionalmente exógena o inelástica a la disponibilidad y uso de cuidados infantiles y su interés radicaba únicamente en que condicionaba la renta del hogar. Pero la crisis económica de 2008 y la evolución de los valores familiares en los padres y madres de niños pequeños nos llevan a prescindir de ese supuesto de exogeneidad. Buscamos indicios de una mayor implicación de los padres en cuidados infantiles que reduzcan la relación entre la demanda de cuidados y la cantidad de horas de que disponen las madres para cuidar de sus hijos pequeños en el hogar. En un trabajo previo (Legazpe y Davia, 2015) se ponía énfasis en la evolución de las decisiones laborales y de cuidados infantiles de las madres durante las diversas fases de la crisis; en éste se hace explícita la relación de dichas decisiones con la situación laboral de los padres y se adopta un periodo de observación algo más amplio (hasta el año 2015).

Nuestros resultados muestran una correlación positiva de los determinantes inobservados que explican tanto la demanda de cuidados formales e informales como la participación laboral de las madres y los padres. Durante la crisis dicha correlación se atenúa en las madres y se intensifica en los padres, lo que podría apuntar a un cierto grado de implicación de aquellos padres que perdieron el empleo en los cuidados de sus hijos pequeños sustituyendo a cuidadores informales esencialmente. También se confirma la sustituibilidad entre la demanda de ambos tipos de cuidados infantiles Y que éste se hace más débil en periodos de crisis que de crecimiento.

Los contenidos de este artículo están organizados del siguiente modo: en primer lugar, presentaremos la evolución de la oferta de cuidados formales y la educación pre-escolar que ha permitido la incorporación de más madres con hijos pequeños al mercado de trabajo español en las últimas dos décadas. En segundo lugar, combinaremos en el marco teórico distintas piezas: por un lado, la oferta laboral femenina y su relación con la demanda de cuidados infantiles; por otro, y dado que los padres pueden sustituir a los cuidados externos dedicando más tiempo a los cuidados en casa, también revisaremos los determinantes de la participación de los padres en el trabajo doméstico y los cuidados

(C) Revista de Economía Laboral 
infantiles en el hogar. Nos interesa en especial formular hipótesis sobre la participación de los padres en los cuidados durante la crisis o cuando atraviesan episodios de desempleo. A continuación, revisaremos la evidencia para el caso español en ambos ámbitos de estudio. Tras una descripción de la base de datos y la muestra, se explica la estrategia empírica multivariante. La discusión de los resultados termina con las conclusiones más relevantes.

\section{Oferta de cuidados infantiles y educación preescolar en España}

A lo largo de las últimas décadas se han desarrollado profundos cambios en el sistema educativo español, en especial en las etapas de educación infantil. Con ello se ha adaptado el sistema a los cambios sociales que se daban en paralelo, como la mayor participación laboral femenina (Llorent, 2013). Como resultado del cambio en el modelo educativo y de la oferta adicional de plazas en centros de educación infantil, la tasa de escolaridad de los menores de tres años ha crecido progresivamente hasta alcanzar niveles superiores a los fijados en los "Objetivos de Barcelona"1.

\footnotetext{
1 En 2002, la Cumbre Europea de Barcelona fijó el llamado 'Objetivo de Barcelona' de prestar para 2010 servicios de educación infantil al 33\% de los niños menores de tres años y al 90\% de los niños en edades comprendidas entre los tres años y la edad de escolarización obligatoria.
} 


\section{Gráfico 1: Alumnado matriculado en educación infantil/preescolar y porcentaje de alumnado matriculado en centros públicos en España (1991/92-2014/15).}

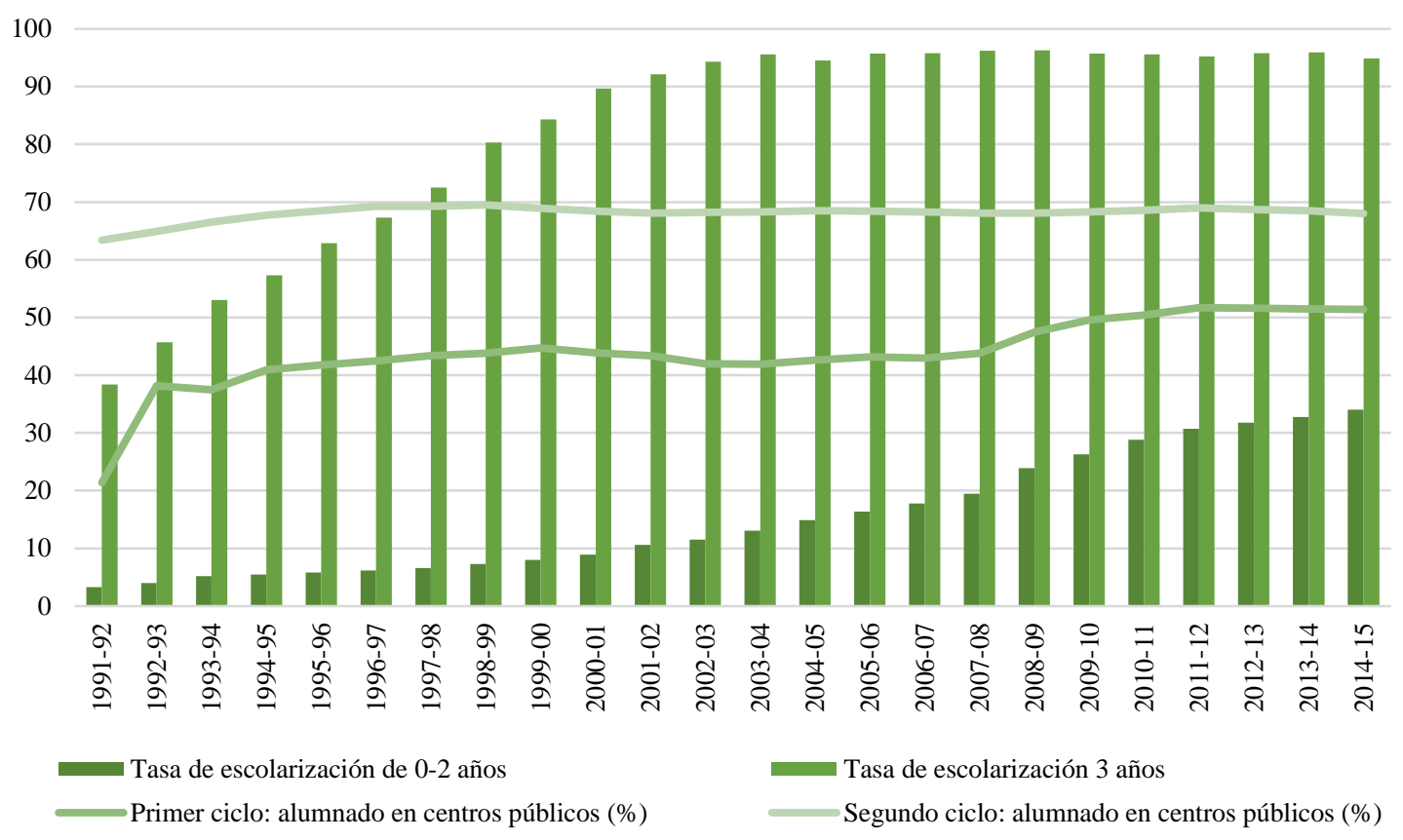

Fuente: Ministerio de Educación, Cultura y Deporte.

De forma similar a lo que ocurre en otros países europeos, en España el sistema de educación infantil se divide en dos ciclos: el primero es para niños menores de tres años y el segundo para los niños de entre tres y cinco años. El porcentaje de plazas de educación infantil en centros públicos para niños menores de tres años ha ido creciendo hasta alcanzar el 50\% y, aproximadamente, dos de cada tres plazas para niños de entre tres y cinco años son públicas y gratuitas. Además, buena parte del tercio restante corresponde a centros concertados cuyas tasas son relativamente asequibles ${ }^{2}$. Esto explica que en este segundo grupo de

2 Indicadores de la $O E C D$ Family Base calculados para dos niños (de dos y tres años de edad) fijaban en 2012 el peso del gasto bruto en cuidados infantiles formales en un $23,7 \%$ del salario medio en España, equivalente a la media de la UE (23,8\%). El coste neto de los cuidados formales a tiempo completo como porcentaje del ingreso neto familiar

(C) Revista de Economía Laboral 
edad las tasas de escolaridad sean casi universales desde el inicio de la década de los 2000 (Gráfico 1).

Las tasas de los centros públicos para niños menores de tres años no distan mucho de las de los concertados y las familias de bajo nivel de renta a menudo tienen prioridad en los centros públicos o reciben algún tipo de subvención. Por todo ello se puede afirmar que las restricciones al uso de cuidados formales y educación pre-escolar vienen más por la disponibilidad de plazas cercanas a la residencia familiar que por el precio (García Román y Cortina, 2016). Ciertamente la distancia en las tasas de escolaridad entre los niños menores de tres y los de tres a cinco años demuestra importantes carencias en la de oferta de cuidados tempranos y educación pre-escolar para niños de muy corta edad, que representa un problema para la participación laboral de muchas madres, quienes se ven obligadas a recurrir a cuidadores informales, a menudo los abuelos, como se verá en la Sección 3.

\section{Marco teórico: más allá del enfoque de capital humano}

En esta sección describimos los enfoques teóricos que han explicado la división del trabajo de mercado y doméstico entre géneros en los hogares, los que predicen la demanda de cuidados infantiles fuera del hogar y los que abordan la distribución del tiempo de cuidados de los hijos en el hogar entre padres y madres convivientes. Terminaremos formulando algunas hipótesis sobre la demanda de cuidados infantiles externos y la distribución de cuidados en el hogar en un contexto de alto desempleo masculino.

\subsection{Oferta laboral de las mujeres y división del trabajo en el hogar}

Economistas y sociólogos han utilizado tres vías principales para explicar la división del trabajo en el seno de los hogares: la primera subraya la búsqueda de la eficiencia productiva a través de la especialización en el hogar (García-Mainar et al., 2011); la segunda busca explicar esta especialización a partir de procesos de negociación intra-

oscilaba entre el 5,6\% en familias de doble ingreso y el 15,1\% en familias monoparental (la media en la UE era de 11,2 y 12,9\%, respectivamente). 
familiar y la tercera pone énfasis en el papel de los valores sociales y aspectos psicológicos, como la identidad.

La vía de la eficiencia viene representada por la Nueva Economía de la Familia (Becker, 1981) según la cual la unidad familiar distribuye su tiempo entre el mercado de trabajo y el trabajo doméstico, de modo que cada miembro de la familia se especialice en la actividad en la que tenga mayor productividad. Este mecanismo de asignación sería, en principio, neutral al género. De acuerdo a su planteamiento básico, la creciente inversión en capital humano y participación laboral de las mujeres en nuestras sociedades deberían haber conducido a una distribución más equitativa del trabajo dentro de los hogares. Pero no ha sido así: en pleno siglo XXI las mujeres españolas asumen un peso mayor en las tareas domésticas que los varones y apenas han reducido el tiempo que dedican al hogar; mientras, aumentan el que dedican al empleo remunerado, algo que sólo pueden conseguir renunciando a tiempo de ocio y de cuidado personal (García Román y Cortina, 2016).

La vía de la negociación surge por lo insatisfactorio que resulta el modelo colectivo (una versión ampliada de la teoría de la eficiencia) para explicar la realidad, pues entiende el hogar como una unidad en la que deben asignarse de forma Pareto-eficiente los tiempos destinados a la producción de bienes y servicios en el hogar y a la producción en el mercado atendiendo a las preferencias de cada miembro. De ahí se ha pasado a otras soluciones inspiradas en la teoría de juegos, tanto no cooperativos como cooperativos (véase un excelente descripción en García Mainar et al., 2011).

En este segundo enfoque es especialmente importante el peso de cada miembro de la pareja en una potencial negociación que establezca la división del trabajo doméstico y extra-doméstico. Una vertiente del mismo plantea una negociación implícita al inicio de la relación en pareja en la que se decide de antemano cómo va a ser esa distribución. Así es cómo Shoshana Grossbard propone en su modelo de diferencias compensatorias la asignación del "trabajo en el hogar" (work-inhousehold). Se trata de un trabajo no remunerado que representa beneficios para el hogar y un coste de oportunidad para quien lo realiza. Cada miembro de la pareja puede compensar al otro para que lo lleve a cabo, "comprando" su tiempo de "trabajo en el hogar". El poder de negociación de cada miembro de la pareja viene dado por la diferencia entre sus ingresos laborales y los de su cónyuge (Grossbard, 2015). Con este criterio, las mujeres asumen más trabajo doméstico que los hombres

C Revista de Economía Laboral 
"compran" con el salario que obtienen en el mercado, superior al de las mujeres. Pero si una mujer tuviera más ingresos que su marido, entonces la asignación sería la contraria. Una vertiente alternativa es la llamada "innovación masculina por necesidad" (González y Jurado-Guerrero, 2009), tradicionalmente conocida como la hipótesis de la disposición de tiempo, por la cual los hombres realizan más tareas domésticas cuando sus mujeres están expuestas a largas jornadas de trabajo.

Pero los hechos no siempre corroboran ni el planteamiento de Grossbard ni la hipótesis de la disposición de tiempo: aunque es cierto que los varones se involucran más cuando las mujeres trabajan largas horas y que las mujeres dedican menos tiempo al trabajo doméstico cuando tienen altos ingresos laborales, ellas mantienen mayor peso relativo en el trabajo en el hogar incluso cuando tienen mayores ingresos que los varones o jornadas laborales más extensas. En estos casos la familia recurre a la externalización del trabajo en el hogar a través de la contratación de servicio doméstico que aligera la carga de trabajo doméstico de ambos miembros de la pareja sin alterar su distribución relativa.

Viendo que también la visión "negociadora" de la asignación del trabajo dentro y fuera del hogar tiene una limitada capacidad explicativa, queda entonces la vía de los valores, que se materializan, a nivel micro, en la identidad y, a nivel macro, en factores estructurales. En el ámbito micro, mujeres y varones no negocian la asignación de las tareas; las llevan a cabo por una mera cuestión de identidad. La identidad femenina se relaciona con las tareas domésticas y la masculina, con la producción fuera del hogar. Cuando los hombres tienen menos peso que sus parejas en la renta del hogar, podríamos decir que se rompe esa relación tradicional entre géneros, esa norma social que marca su identidad (Coltrane, 2000). Entonces puede haber una reacción por parte de uno de los miembros de la pareja (o de ambos) hacia comportamientos tradicionales: los varones, para reforzar su masculinidad, realizarán menos tareas domésticas y las mujeres, para compensarles por esta situación anormal, asumirán aún más trabajo en el ámbito del hogar (Bittman et al., 2003). La perspectiva sociológica que formula este planteamiento se conoce como construcción simbólica de género (doing gender), por la cual "la división del trabajo es principalmente una práctica social que se crea y reconstruye por las mujeres y los hombres, como también a través de instituciones sociales como la familia, el Estado de bienestar y el mercado laboral" (González y Jurado-Guerrero, 2009; p. $67)$. 
Tal y como se recoge en Sevilla-Sanz et al. (2010), este concepto se sostiene en la idea de que los individuos internalizan las expectativas de género que sobre ellos tienen los demás y actúan en consecuencia. Su equivalente en economía es la Economía de la Identidad, de Akerlof y Kranton (2000). Estos autores incorporan en sus modelos la psicología y la sociología de la identidad (la autopercepción) como un elemento determinante en el comportamiento de los individuos asociado a categorías y normas sociales. Un esposo puede perder identidad (y ver reducida así su utilidad individual) cuando su mujer tiene mayores ingresos que él, lo que la lleva a asumir una mayor cuota del trabajo doméstico en el hogar (y no menos, como sería de esperar dado su mayor poder de negociación) para reestablecer esta utilidad perdida del varón.

En el ámbito macro, la capacidad de negociación de las mujeres queda anulada por factores externos como las normas no escritas, las instituciones y la cultura. Algunas contribuciones feministas explican que el contexto social, las normas sociales, los principios éticos, las relaciones patriarcales y marcadas por el género, las regulaciones, instituciones, legislación y políticas públicas van a marcar la asignación del trabajo dentro del hogar (Berik y Kongar, 2013). Son elementos estructurales ante los cuales las mujeres no tienen capacidad de acción ni de negociación y que perpetúan un reparto desequilibrado e ineficiente de la asignación del tiempo de trabajo fuera y dentro del hogar.

\subsection{Demanda de cuidados infantiles}

Para poder compatibilizar trabajo y familia es necesario "comprar tiempo" para el mercado a través de servicios de cuidados infantiles. La forma tradicional de explicar la demanda de cuidados infantiles viene inicialmente dada por la teoría convencional del consumidor: los padres demandan cuidados infantiles en el mercado de acuerdo a sus preferencias y restricciones tanto presupuestarias como temporales (en Chaudry et al. (2010) se hace una profunda revisión de esta literatura). Las preferencias de los padres están relacionadas con su nivel educativo, siendo aquellos que han alcanzado un mayor nivel quienes mejor valoran los beneficios de la educación a edades tempranas, lo que les lleva con mayor frecuencia a demandar cuidados formales en centros educativos. Las restricciones presupuestarias vienen dadas por la renta del hogar y

(C) Revista de Economía Laboral 
las temporales, por la situación laboral de las madres esencialmente (García-Mainar et al., 2011).

La decisión de utilizar cuidados infantiles conlleva también la elección del tipo de cuidados. Ésta a su vez depende (de nuevo) de las preferencias, así como de los precios relativos y el número de niños en el hogar. Los padres con mayor nivel de estudios tienden a preferir los cuidados formales en la medida en que favorecen la socialización y la estimulación temprana de los niños (Del Boca y Vuri, 2007; Mamolo et al., 2011). Los precios relativos marcan también la relación de sustituibilidad de los cuidados formales e informales y la disponibilidad de centros infantiles cercanos al hogar o vivir cerca de los familiares también marca las decisiones de los padres y madres (Borra, 2010). Por último, para el hogar es más eficiente el uso de una única forma de cuidados cuando hay varios niños en edad pre-escolar; en tales casos es más probable optar por los cuidados formales, al resultar más difícil conseguir ayuda informal en el entorno familiar para dos o más niños que para uno solo (Del Boca y Vuri, 2007).

Un enfoque exclusivamente de consumo olvida que los padres no sólo satisfacen su utilidad como consumidores de educación pre-escolar con los cuidados, sino que también "compran" con ello tiempo que pueden dedicar al mercado de trabajo y que, por tanto, esta demanda está íntimamente relacionada con la oferta laboral de las madres (Chaudry et al., 2010). El reconocimiento de esta relación endógena entre ambas variables lleva a la determinación simultánea de la demanda de cuidados y la participación laboral femenina (partiendo de Connelly (1992) y Ribar (1995) se ha abierto una amplia línea de evidencia empírica con múltiples ejemplos como Chiuri (2000); Del Boca y Vuri (2007); Powell (2002); Tekin (2007); Van Gameren y Ooms (2009), entre otros).

Además, aplicar la teoría del consumidor a la demanda de cuidados infantiles supone no preguntarse hasta qué punto el cuidado de los hijos es una tarea doméstica y externalizable. Los cuidados infantiles comparten características con el ocio (son agradables y pueden tener un componente lúdico y social, que aumenta la reputación de los padres como tales) y con las tareas domésticas (requieren de mucho tiempo no remunerado, lo que implica aceptar un coste de oportunidad), sin llegar a ser ni lo uno ni lo otro (Ribar, 1995) porque no tienen fácil sustituto en el mercado. Hay tareas que los padres no pueden o incluso no están dispuestos a externalizar. El tiempo que pasan con sus hijos contribuye a su identidad como padres y les genera utilidad. Hay ámbitos de los cuidados, como los más relacionados con la educación, a los que los padres 
y madres no renuncian independientemente de sus restricciones temporales (Pailhe y Solaz, 2008).

(C) Revista de Economía Laboral 


\subsection{Ciclo económico, oferta laboral de los padres y uso de cuidados infantiles}

El enfoque de consumo predice que la experiencia del desempleo durante una crisis económica aplicado a la demanda de cuidados infantiles fuera del hogar disminuirá la demanda de cuidados infantiles en el mercado como resultado de la caída en la renta disponible del hogar y aumentará la cantidad de tiempo que los padres dediquen a cuidar de sus hijos en casa (Aguiar et al., 2011; Morrill y Pabilonia, 2015). Por otro lado, la pérdida de ingresos en la unidad familiar puede llevar a algunas madres inactivas a ofrecer su tiempo en el mercado de trabajo (efecto trabajador añadido). Se plantea entonces más disponibilidad de tiempo en los varones y menos en las mujeres ¿Cómo se distribuirá el nuevo tiempo de cuidados en el hogar entre padres y madres? Un enfoque de negociación predice que la pérdida de poder negociador hará que los padres asuman más tareas domésticas y de cuidados, pero una perspectiva de "doing gender" - o de identidad - apuntará justo a lo contrario: en las crisis las mujeres pueden terminar aportando aún más trabajo en el hogar, renunciando a ocio y tiempo personal para compensar a los varones por la ruptura de la norma social por la cual ellos aportan renta al hogar y no tareas domésticas (Bittman et al., 2003). La respuesta sólo puede venir de la evidencia empírica.

\section{Evidencia empírica: el caso español}

\subsection{Demanda de cuidados infantiles y oferta laboral de las madres}

La evidencia sobre cuidados infantiles y su relación con la oferta laboral de las madres ha recibido una notable atención en la literatura internacional (un excelente survey sobre el tema es Morrissey, 2017). En general se encuentra que los determinantes de la demanda de cuidados (costes y disponibilidad) también contribuyen a explicar la oferta laboral de las madres. De igual modo, es un resultado muy habitual que la renta y composición del hogar y, en especial, el nivel educativo de los padres, son importantes factores que explican el tipo de cuidados infantiles que las familias demandan. En España la importancia de los costes de los cuidados viene demostrada en Borra (2010) mientras que la disponibilidad de cuidados se muestra también relevante en Baizán y González (2007) y Suárez (2013). 
Dado que la educación pre-escolar es prácticamente universal a partir de los tres años de edad, la mayor parte de la evidencia de cuidados infantiles en España se centra en los niños menores de tres años y es más abundante al respecto de los cuidados formales que de los informales, para los que la información es más escasa. Así, en Borra (2010) se subraya el papel del precio de los cuidados en la determinación tanto de su demanda como de la participación laboral de las madres, y en un trabajo previo (Borra y Palma, 2009) se prestaba en cambio más atención a la disponibilidad de centros de cuidados en el entorno del hogar y a sus necesidades. Las autoras encuentran además una relación de sustituibilidad entre cuidados formales e informales y, a su vez, entre cuidados formales y parentales, marcada por la evolución de sus precios relativos. También la elección entre cuidados formales e informales de mercado frente a los gratuitos es analizada en Suárez (2013), donde se comprueba la importancia de la educación de los padres, la composición y la renta del hogar, así como algunas características del empleo de las madres en la elección del tipo de cuidados infantiles que demandan.

\subsection{Los cuidados en el hogar y su distribución entre los miembros de la pareja}

En España el tiempo dedicado a tareas estrictamente domésticas tiene una asignación muy desigual por sexos, que va más allá de la que se esperaría de las características personales y laborales de los miembros de la pareja y, por tanto, tiene un importante componente de "cultura de género" (Álvarez y Miles, 2003). Esto responde al peso importante de los valores tradicionales de género en la sociedad española (Sevilla-Sanz et al., 2010). De hecho, resultados obtenidos de encuestas de uso del tiempo en España recogen lentos avances en la convergencia entre cónyuges en el tiempo que dedican a las tareas domésticas y a los cuidados de los hijos en el hogar (Rodríguez y Ugidos, 2017), si bien es cierto que aumenta la proporción de varones que participa en ambas tareas. En efecto, la implicación de los padres españoles en los cuidados es creciente y se advierte sobre todo en las parejas de doble ingreso, aunque responda más a los cambios en la composición laboral y educativa de las madres que en los valores de género (Ajenjo y García-Román, 2014). También está muy relacionado con el nivel educativo de los padres (Gutiérrez-Domènech, 2010; Gimenez-Nadal y Molina, 2013; Gracia, 2014) y recientemente se describen nuevas formas de paternidad o paternidades emergentes

(C) Revista de Economía Laboral 
(Barbeta-Viñas y Cano, 2017; García-Román y Cortina, 2016). De todos modos, las madres siguen teniendo un mayor protagonismo que los padres en los cuidados infantiles (Meil y Rogero-García, 2015). Los cuidados de los padres son más bien suplementarios a los de las madres, en especial cuando éstas tienen largas jornadas laborales ${ }^{3}$. En el caso de los cuidados rutinarios, más intensivos en tiempo, la implicación de los padres responde más a la situación laboral y a la jornada de las madres que a la suya propia (Gracia y Esping-Andersen, 2015).

Por otro lado, muchas familias recurren a los abuelos para el cuidado de los niños fuera del horario escolar e incluso el cuidado extensivo durante toda la jornada laboral. Y aunque el apoyo de los abuelos contribuye a la participación laboral de las madres, en GarcíaMorán y Kuehn (2012) se advierte de que el cuidado regular por parte de los abuelos limita la movilidad residencial y geográfica de las madres y, con ello, también sus oportunidades laborales y salariales.

Respecto a las horas de cuidados en el hogar, en García-Mainar et al. (2011) se advierte que apenas responden a la jornada laboral, seguramente por lo inelástica que es. Por otro lado, el peso de las madres en la renta del hogar está negativamente correlacionado con el tiempoabsoluto - que dedican a los cuidados mientras que la renta de las familias en su conjunto se relaciona positivamente con la cantidad de tiempo que los padres pasan con sus hijos, lo que sugiere que los cuidados infantiles tienen un importante componente de inversión a largo plazo en capital humano y otro de atención y de disfrute por parte de los padres, que no es externalizable y al que no renuncian, independientemente del nivel de renta. De todos modos, en el caso de cuidados de niños muy pequeños, como los que analizamos en este trabajo, en Gracia (2014) se detecta que la duración de la jornada del padre limita su implicación en los cuidados y que ésta a su vez ésta responde a la duración de la jornada de las madres.

En cuanto a qué ocurre con la distribución del tiempo de cuidados en casa cuando uno de los miembros de la pareja pierde su empleo, no hemos encontrado evidencia directa para el caso español, pero la de países de nuestro entorno nos podría dar algunas pistas. En Pailhe y

\footnotetext{
${ }^{3}$ Además de las largas jornadas laborales hay un hecho diferencial en la organización del tiempo de trabajo en España que ha demostrado ser un problema particularmente grave para la conciliación laboral y familiar y la atención a los hijos: la jornada partida, habitual en muchas empresas (García-Román y Cortina, 2016; Gutiérrez-Domènech, 2010; Gracia y Kalmijn, 2016).
} 
Solaz (2008) se analiza cómo la situación de desempleo de uno de los padres supone una reorganización de los tiempos de cuidados dentro de la pareja en Francia. Los autores advierten una sustitución imperfecta de las actividades de cuidados dentro de la pareja puesto que el progenitor ocupado cede al que está en desempleo tareas rutinarias, como el transporte, pero no las educativas y de ocio. Por otro lado, tampoco es simétrica la cantidad de tiempo el progenitor en desempleo "libera" al que está ocupado: cuando la madre es la que se encuentra en desempleo "libera" de más horas de cuidados al padre ocupado que cuando es el padre el que se encuentra en paro mientras la esposa permanece ocupada. Esto último es algo que ocurre en general con las tareas domésticas: en Van der Lippe et al. (2017) se analiza una muestra de 28 países con la Encuesta Social Europea en 2004 y 2010 y se advierte que cuando las mujeres caen en el desempleo tienden a involucrarse más en las tareas domésticas que lo que lo hacen los varones que pierden su trabajo. Es más, cuando son los varones quienes pierden su trabajo, las mujeres ocupadas también reaccionan aumentando el tiempo que dedican a las tareas del hogar.

\section{Base de datos y muestra seleccionada}

Los datos utilizados en este artículo proceden de la Encuesta de Condiciones de Vida (en adelante, ECV). Dirigida a todo tipo de hogares residentes, su diseño responde a directrices europeas, pues forma parte de las European Statistics on Income and Living Conditions (EU-SILC) coordinadas por Eurostat. La información sobre horas de trabajo de los padres y de cuidados infantiles a cargo de terceras personas proporcionan información suficiente para contrastar hipótesis sobre la relación entre las decisiones de cuidados infantiles y las características de los padres, del hogar y del entorno, pero no sobre la cantidad de horas de cuidados en el hogar ni su distribución en el seno de la pareja, sobre las que sólo podremos formular hipótesis fundamentadas. En este trabajo explotamos los ficheros transversales de la ECV entre 2005 y 2015, analizando una muestra que está compuesta por 7.924 observaciones de niños menores de tres años ${ }^{4}$ enlazados a los cuestionarios de sus madres y de sus

${ }^{4}$ La edad máxima de los niños en la muestra es de 42 meses. De lo contrario, en el momento de la entrevista (entre enero y junio de cada año) ya asistirían a centros de educación preescolar. En España, a partir de esa edad, la tasa neta de matriculación en

(C) Revista de Economía Laboral 
respectivas parejas - varones - convivientes $^{5}$ (y que se corresponden, en total, con 6.602 hogares biparentales). Es decir, a diferencia de la estrategia habitual de la literatura, que contempla muestras conformadas por mujeres a las que se le vinculan características de sus hijos, en la muestra aquí utilizada las observaciones se corresponden con los niños y las de las madres y los padres se repiten tantas veces como hijos menores de tres años tengan, dado que cada uno de ellos puede estar recibiendo cuidados de distinto tipo en el momento de la entrevista. De todos modos, apenas el 15\% de las observaciones, corresponden a hogares donde hay más de un niño menor de tres años (independientemente de que también haya niños mayores de esa edad) y por tanto se encuentran duplicadas.

La Tabla 1 muestra la incidencia de las cuatro variables de interés (la madre trabaja, la pareja trabaja, uso de cuidados formales y uso de cuidados informales): casi el $61 \%$ de las observaciones corresponden a niños cuyas madres tienen un trabajo remunerado, y más del 88\% corresponden a padres ocupados. El 43\% de los niños de la muestra reciben cuidados formales y sólo el $21 \%$ recibe cuidados informales. La mayor parte de los niños que reciben cuidados formales asisten a centros de preescolar, mientras que los cuidados informales son proporcionados en su gran mayoría por personas que no obtienen ninguna remuneración por ello (abuelos, otros parientes, amigos, vecinos, etc.).

educación preescolar es casi universal y, por tanto, la decisión de utilizar cuidados formales ya no es relevante en nuestro estudio.

${ }^{5}$ En algunos casos las parejas de las madres pueden no ser los padres biológicos de los niños de la muestra. Dada la corta edad de los niños de la muestra esto es poco probable y, además, para nuestros efectos entendemos que no es relevante, puesto que es la convivencia y no la paternidad en sí la que puede marcar la distribución intrafamiliar de trabajo remunerado y no remunerado y cuidados en el hogar. 
Tabla 1: Incidencia de las variables dependientes (\%).

\begin{tabular}{|c|c|c|c|c|c|c|}
\hline & \multicolumn{2}{|c|}{$\begin{array}{c}\text { Periodo } \\
\text { crecimiento } \\
(2005-2008 \\
\text { 2015) } \\
\end{array}$} & \multicolumn{2}{|c|}{$\begin{array}{l}\text { Periodo crisis } \\
(2009-2014)\end{array}$} & \multicolumn{2}{|c|}{ Total } \\
\hline \multicolumn{7}{|c|}{ Incidencia de las variables dependientes (\%) } \\
\hline Mujer trabaja & \multicolumn{2}{|c|}{58,33} & \multicolumn{2}{|c|}{63,25} & \multicolumn{2}{|c|}{60,76} \\
\hline Pareja trabaja & \multicolumn{2}{|c|}{93,45} & \multicolumn{2}{|c|}{83,07} & \multicolumn{2}{|c|}{88,31} \\
\hline Cuidados formales* & \multicolumn{2}{|c|}{42,74} & \multicolumn{2}{|c|}{43,59} & \multicolumn{2}{|c|}{43,16} \\
\hline Preescolar & \multicolumn{2}{|c|}{39,80} & \multicolumn{2}{|c|}{42,41} & \multicolumn{2}{|c|}{41,09} \\
\hline Centro fuera horario escolar & \multicolumn{2}{|c|}{0,40} & \multicolumn{2}{|c|}{0,00} & \multicolumn{2}{|c|}{0,20} \\
\hline Otros centros & \multicolumn{2}{|c|}{3,02} & \multicolumn{2}{|c|}{1,38} & \multicolumn{2}{|c|}{2,21} \\
\hline Cuidados informales* & \multicolumn{2}{|c|}{23,78} & \multicolumn{2}{|c|}{19,05} & \multicolumn{2}{|c|}{21,44} \\
\hline Cuidadores profesionales & \multicolumn{2}{|c|}{4,42} & \multicolumn{2}{|c|}{2,55} & \multicolumn{2}{|c|}{3,50} \\
\hline Otras personas sin remunerar & \multicolumn{2}{|c|}{20,01} & \multicolumn{2}{|c|}{16,81} & \multicolumn{2}{|c|}{18,43} \\
\hline \multicolumn{7}{|c|}{ Número de horas en cada situación (cuando son mayores que cero) } \\
\hline & media & d.s. & media & d.s. & media & d.s. \\
\hline Mujer trabaja & 34,6 & 9,2 & 34,6 & 9,2 & 34,6 & 9,2 \\
\hline Pareja trabaja & 43,3 & 8,0 & 42,4 & 7,4 & 42,9 & 7,7 \\
\hline Cuidados formales* & 26,6 & 10,8 & 27,0 & 10,3 & 26,8 & 10,5 \\
\hline Preescolar & 26,7 & 11,0 & 27,1 & 10,2 & 26,9 & 10,5 \\
\hline Centro fuera horario escolar & 8,1 & 5,8 & 0,0 & - & 8,1 & 5,8 \\
\hline Otros centros & 24,4 & 13,2 & 20,6 & 9,9 & 23,0 & 12,2 \\
\hline Cuidados informales* & 23,4 & 14,6 & 24,9 & 13,8 & 24,1 & 14,2 \\
\hline Cuidadores profesionales & 24,1 & 14,9 & 26,8 & 13,3 & 25,2 & 14,3 \\
\hline Otras personas sin remunerar & 23,0 & 15,3 & 24,5 & 14,1 & 23,8 & 14,7 \\
\hline Número de observaciones & \multicolumn{2}{|c|}{4.003} & \multicolumn{2}{|c|}{3.921} & & \\
\hline
\end{tabular}

Fuente: Encuesta de Condiciones de Vida. Ficheros transversales 2005-2015. Instituto Nacional de Estadística.

Hemos separado la muestra en dos periodos que hemos diferenciado, esencialmente, por el nivel de empleo de los padres. En el periodo 2009-2014 (crisis) las tasas de ocupación de los padres eran diez puntos porcentuales inferiores a los años previos a la crisis (2005-2008), a los que hemos sumado el de recuperación ${ }^{6}$ (2015). Juntas, las

\footnotetext{
${ }^{6}$ El año 2015 es un año de recuperación en el mercado de trabajo español, por lo que lo clasificamos en el periodo de "crecimiento". En Izquierdo y Regil (2017) puede advertirse que en ese año se registra un crecimiento interanual de los niveles de empleo claramente
}

(C) Revista de Economía Laboral 
observaciones correspondientes a los periodos 2005-2008 y el año 2015 conforman una sub-muestra que etiquetaremos como "crecimiento" en contraste con la etiqueta "crisis". Durante la crisis la tasa de ocupación de las madres aumentó en cinco puntos porcentuales. Y aunque la incidencia de los cuidados formales casi no cambia, en los informales se aprecia una importante caída (cinco puntos porcentuales) en las épocas de baja ocupación masculina, sobre todo en los que proporcionan cuidadores no remunerados. Nuestra intuición es que la caída de cuidados informales viene dada por una mayor implicación de los padres en los cuidados en el hogar y que las familias no renuncian a los cuidados formales aunque caiga su nivel de renta, dado su componente educativo y su asequibilidad.

En cuanto a la cantidad de horas dedicadas a trabajo y cuidados (cuando son mayores que cero) encontramos que las jornadas laborales de padres y madres son relativamente largas, en especial las de los padres, y que los cuidados formales cubren un rango de horas algo más largo - y menos disperso - que los cuidados informales. Además, es interesante lo poco que oscilan las jornadas laborales y de cuidados entre los dos sub-periodos como resultado de la escasa flexibilidad de las jornadas laborales en España, que llevan también a una escasa variación en las horas de cuidados demandadas. Los ajustes ante la crisis se producen, por tanto, en la incidencia del empleo y de los cuidados, y no en la duración de las jornadas laborales ni en la cantidad de horas demandadas de cuidados infantiles no parentales.

\section{Metodología - modelo tobit multivariado}

La estrategia econométrica que desarrollamos aquí, un modelo tobit multivariado, permitirá estimar el tiempo semanal que las madres y sus parejas, cuyo rol en el hogar identificaremos con el del padre, dedican el trabajo remunerado, así como la cantidad de horas a la semana de cuidados formales e informales que utilizan para cada uno de sus hijos menores de tres años. La forma funcional (tobit) permite tener en cuenta la distribución de las variables dependientes, censuradas en cero en el

positivo en todos los trimestres (del 3\% de media). Además, empieza en ese año a caer el desempleo de larga duración (la tasa de paro general ya había caído en 2014 pero en parte como resultado de la caída de la actividad, mientras que en 2015 respondió prácticamente solo al incremento de la ocupación). 
caso de padres y/o madres no ocupados y/o que no demandan algún tipo de cuidados para sus hijos. Además, la estructura multivariante permite considerar relaciones endógenas entre las variables de interés resultado de la influencia de variables inobservadas que las explican simultáneamente, como los valores e identidad de género, las relaciones intrafamiliares y la disponibilidad de cuidados formales e informales en el entorno de la vivienda, entre otros.

El planteamiento de los modelos parte de cuatro variables latentes, $y_{i 1}{ }^{*}, y_{i 2}{ }^{*}, y_{i 3}{ }^{*}$ y $y_{i 4}{ }^{*}$, que representan la utilidad de las horas de trabajo de la madre, de la pareja y de cuidados formales e informales, respectivamente (Wooldridge, 2010):

$$
\begin{aligned}
& y_{m}{ }^{*}=B_{m}{ }^{\prime} X_{m}+\varepsilon_{m}, \quad m=1,2,3,4 \\
& y_{m}=\max \left(0, y_{m}{ }^{*}\right)
\end{aligned}
$$

donde $X_{m}(m=1,2,3,4)$ son los $k \times 1$ vectores de las variables explicativas observables, $B_{m}(m=1,2,3,4)$ son los vectores de los coeficientes desconocidos a estimar; $\mathcal{E}_{m}$ son los términos de error, que siguen una distribución normal multivariante $(\mathrm{N}(0, \Sigma))$, con media cero y caracterizados por una matriz de covarianzas $(\Sigma)$ que recoge los coeficientes de correlación entre los términos de error de las ecuaciones estructurales, potencialmente distintas de cero fuera de la diagonal principal $\left(\rho_{12}, \rho_{13}, \rho_{14}, \rho_{23}, \rho_{24}, \rho_{34}\right)$ debido a factores no observables que afectan a las variables dependientes. Si dichas correlaciones son significativas, ello significará que los estimadores son más eficientes y tienen menos riesgo de sesgo que los que se obtendrían estimando las cuatro ecuaciones por separado. El signo de las correlaciones estimadas confirmará si las decisiones de participación de las madres y de los padres y las decisiones de cuidados formales e informales son complementarias (cuando la correlación positiva) o sustitutivas (cuando es negativa). Para estimar el modelo se ha utilizado el comando $\mathrm{cmp}$ de Stata creado por Roodman (2011).

En la determinación del modelo los vectores de variables exógenas de las cuatro ecuaciones describen cuatro ámbitos: (a) características sociodemográficas (edad y nivel educativo de la madre); (b) la composición familiar (edad del niño, presencia de otros hijos en el hogar y situación laboral de la pareja); (c) características económicas del hogar (receptor de

(C) Revista de Economía Laboral 
ayuda por familia/hijos y renta del hogar neta de la madre) y (d) características del entorno (región (NUT1) de residencia, grado de urbanización y tasa de paro de la CCAA, por sexo). Adicionalmente, se incorpora como última variable de control el año de la entrevista.

Esperamos confirmar el perfil habitual de la oferta laboral de las madres y de la demanda de cuidados. Nuestro interés se centra, por un lado, en la relación entre las horas trabajadas por padres y madres y el uso de cuidados infantiles y, por otro lado, la reacción de la demanda de cuidados al desempleo de los padres. Pretendemos así comprobar si pérdida del empleo en los varones se corresponde con una menor correlación entre las horas de cuidados fuera del hogar y la situación laboral del padre ${ }^{7}$ durante la crisis, lo que podría ser un síntoma de una cierta sustitución de cuidados informales por los proporcionados por el padre en el hogar.

Los valores medios de todas las variables incluidas en el modelo multivariante se muestran en la Tabla A.1 del Apéndice. En ella puede observarse que la edad media de las madres es de casi 35 años y que tienen un mayor nivel educativo que sus parejas (51\% de ellas tienen estudios universitarios, ocho puntos por encima de los cónyuges). El 46\% de las observaciones corresponden a hijos únicos. Uno de cada tres niños de la muestra vive en hogares que reciben alguna ayuda o prestación por hijos y casi la mitad reside en entornos urbanos, densamente poblados. Entre los dos sub-periodos identificados en la Tabla 1 apenas se producen cambios salvo por la mayor incidencia de paro e inactividad de las parejas en el periodo 2009-2014.

\section{Resultados - discusión}

En la Tabla 2 aparecen los resultados de los modelos multivariantes expresados como efectos marginales, que representan la variación en el número de horas semanales de las cuatro actividades analizadas cuando cada las variables explicativas aumentan en una unidad.

\footnotetext{
${ }^{7}$ En la estimación de las horas de cuidados fuera del hogar se hace explícita la situación laboral el padre pero no la de la madre porque la situación laboral de esta última está muy correlacionada con varias de las variables explicativas que aparecen en la ecuación (su nivel educativo, su edad, la presencia de otros niños en el hogar y sus edades, así como la del propio niño que representa cada observación).
} 
La participación laboral de las madres sigue una evolución en forma de $\mathrm{U}$ invertida con la edad, lo que supone un patrón inverso al de los varones (en forma de U). Las horas destinadas al mercado de trabajo de ambos cónyuges se relacionan positivamente con el nivel educativo, si bien en el caso de los varones están menos correlacionadas con el hecho de tener educación universitaria que en el de las mujeres.

En cuanto a la demanda de cuidados no parentales, se confirma nuestra primera hipótesis acerca de la mayor respuesta de la participación laboral de las madres a la presencia de hijos en el hogar y a las edades los niños que la de los padres: las madres que tienen más de un hijo presentan menos horas de trabajo, mientras que los varones sólo dedican menos tiempo al mercado si conviven con niños tanto mayores como menores de tres años. A su vez, cuando hay más de un niño en el hogar se recurre más a cuidados formales y menos a los informales, son más sencillos de conseguir para hijos únicos.

Siguiendo el patrón esperado en el marco neoclásico tradicional, la renta del hogar guarda la esperada relación negativa con las horas de trabajo de la mujer y positiva con la demanda de cuidados, sobre todo los formales. En cambio, la percepción de ayudas por hijos a cargo tiene una notable correlación positiva con ambas variables, puesto que entre sus objetivos está incrementar la participación laboral de madres que no aspirarían a salarios elevados en el mercado.

Para un nivel dado de renta familiar, el desempleo del padre queda negativamente correlacionado con la demanda de cuidados externos, sobre todo de cuidados informales, que son más fácilmente sustituibles por el cuidado paterno en el hogar. En las zonas rurales y de densidad poblacional intermedia hay menos empleo femenino que en las urbanas y en ella son menos habituales los cuidados informales y se usan un poco más los formales, si bien las diferencias entre distintos territorios no son muy pronunciadas. El empleo femenino alcanza valores máximos en la Comunidad de Madrid y en las regiones del Este (Cataluña, Comunidad Valenciana e Islas Baleares); pero mientras en la Comunidad de Madrid las madres afrontan los altos niveles de empleo con cuidados formales, en otras regiones, en especial en la región Este, se utilizan más los informales.

La evolución temporal y del ciclo marcan también la tendencia observada, con un número creciente de horas de trabajo de las madres a partir del año 2008, avances en cuidados formales (salvo en 2012 y 2015)

(C) Revista de Economía Laboral 
y retroceso continuado en cuidados informales, especialmente importante en 2012 y 2013 - coincidiendo con los niveles mínimos de empleo de los padres - y 2015 respecto de la situación inicial. En cuanto a nuestra segunda hipótesis, no se percibe el efecto "trabajador añadido" en las madres ante situaciones de desempleo en sus parejas; antes al contrario, se advierte una correlación positiva entre la cantidad de horas de trabajo de ambos miembros de la pareja, siendo más intensa la correlación cuando se estima la cantidad de horas de trabajo en el padre a partir de la situación laboral de la madre que cuando se analiza la relación en dirección contraria.

Los términos de error guardan las correlaciones bilaterales esperadas: la demanda de cuidados tanto formales como informales viene explicada por factores inobservados que también condicionan, y en el mismo sentido, la oferta laboral de padres y madres, si bien estas correlaciones son más intensas cuando se trata de la participación laboral de las madres que de los padres y también en los cuidados informales que en los formales. A su vez, los cuidados formales y los informales son sustitutivos entre sí, al estar determinados por factores no observables que cuando inducen a utilizar los de un tipo, reducen el uso del otro.

Con el objetivo de comprobar la tercera hipótesis de este trabajo (cambio, durante la crisis, en la relación entre la oferta laboral de ambos cónyuges y menor sensibilidad de la demanda de cuidados a las horas de trabajo de las madres ante una posible implicación de los padres en sus cuidados) estimamos los modelos para los dos sub-periodos identificados en la Tabla 1 por separado (Tabla A.2). Por razones de espacio sólo presentamos los efectos marginales de las variables más relacionadas con esta tercera hipótesis, así como los coeficientes de correlación entre los términos de error: en la crisis la situación laboral de las madres guarda un correlación bastante menor con la de sus parejas que en la época de crecimiento, quizá como resultado de la naturaleza asimétrica de la pérdida de empleo durante la recesión, muy concentrada en sectores masculinizados. Al mismo tiempo, cambia el signo de la correlación entre los términos de error de las ecuaciones de participación laboral de los cónyuges, pasando de negativo en el periodo crecimiento a positivo durante la crisis: quizá los valores tradicionales y la identidad de género que en tiempos de bonanza llevan a las mujeres a participar menos en el mercado se convierten en un compromiso con la actividad económica fuera del hogar para completar las rentas familiares, sensiblemente mermadas durante la crisis, sin que eso merme su aportación al trabajo doméstico, inobservable con la base de datos utilizada. 
La segunda parte de nuestra tercera hipótesis se refiere a los posibles cambios en la sensibilidad de la demanda de cuidados formales y, sobre todo, informales en los periodos de baja ocupación masculina. El cambio de ciclo no alteran la relevancia de las variables inobservadas que explican el empleo de las madres y su uso de cuidados formales e informales, pero sí las relacionadas con los padres y la demanda de cuidados informales. Simultáneamente disminuye la relación de sustituibilidad entre ambos tipos de cuidados. Todo ello podría apuntar a una mayor implicación de los padres en los cuidados de los hijos cuando pierden su empleo, sustituyendo esencialmente a cuidadores informales.

La evidencia obtenida no corrobora la hipótesis de identidad de Akerlof y Kranton (2010) y parece más bien ajustarse a los postulados de enfoques previos. El hecho de que cuando los padres se encuentran en desempleo caiga la demanda de cuidados tanto formales como, sobre todo, informales mientras las madres aumentan su tiempo en el mercado laboral y ven más desvinculada su situación laboral de la de sus cónyuges estaría en línea con postulados en el ámbito de la negociación intrahogar. Pero sin una adecuada observación de la asignación de los tiempos de cuidados en el hogar entre padres y madres no será posible afirmarlo con contundencia.

\section{Conclusiones}

En este trabajo hemos analizado los patrones de participación laboral de madres y padres de niños pequeños y su vinculación con la demanda de cuidados formales e informales para comprobar sus posibles especificidades en épocas de alto desempleo de los padres y asimetrías en el compromiso de padres y madres con los cuidados durante los episodios de desempleo. Una estimación simultánea del tiempo semanal que dedican padres y madres al trabajo remunerado y que pasan en cuidados formales e informales por los hijos menores de tres años, sobre una muestra extraída de la Encuesta de Condiciones de Vida (ECV) en el periodo 2005-2015, confirma la tradicional correlación positiva entre la participación laboral de padres y madres y la demanda de cuidados formales e informales. Sin embargo, ésta se suaviza en las madres y se incrementa para los padres durante la crisis, lo que podría indicar cierta implicación de los padres que perdieron el empleo en los cuidados de sus hijos pequeños sustituyendo, esencialmente, a cuidadores informales.

(C) Revista de Economía Laboral 
Para poder corroborar esto último necesitaríamos superar la carencia más relevante de nuestra base de datos: al no ser una encuesta de uso del tiempo, la información sobre actividades cotidianas está muy limitada y no se dispone de datos que permitan corroborar directamente la correspondencia entre un menor número de horas de trabajo en el mercado con un mayor número de horas de cuidados infantiles en casa. Sólo a través de los cambios en las correlaciones observadas entre las jornadas laborales y el tiempo de cuidados no parentales tenemos una evidencia (siempre indirecta) de estas posibles relaciones, eso sí, en la dirección esperada. Una posible línea de investigación futura consistiría en contrastar las tendencias aquí indirectamente observadas con encuestas que contemplen el uso del tiempo en distintas actividades en los hogares españoles. Pero, aunque de este modo se podría permitir corroborar cambios en los patrones de uso del tiempo de los varones en función de su situación laboral, difícilmente se podría adoptar una perspectiva temporal que permitiese observar la evolución del ciclo económico y cómo conforme se prolonga la situación de desempleo de los padres se van involucrando en los cuidados. Además, mientras la ECV carece de información sobre la distribución del tiempo de cuidados de padres y madres en el hogar, las bases de datos provistas de información sobre usos del tiempo carecen de información sobre cuidados fuera del hogar, ya sean formales o informales. Por tanto, con ninguna de las fuentes de información sería posible ofrecer una descripción que implique alcanzar una descripción de la oferta laboral de padres y madres a lo largo del ciclo, la demanda de los cuidados externos y la provisión de cuidados paternales en el seno del hogar.

Con estas historias fragmentadas creemos haber contribuido a la literatura sobre cuidados externos y participación laboral de las madres comprobando que incorporar al tercer agente (los padres) en el análisis, contribuye a entender el comportamiento éstas en el mercado de trabajo, y que en situaciones de alto y prolongado desempleo están contribuyendo a una "des-feminización" de los patrones de participación laboral y demanda de cuidados. Ahora resta ver si en el futuro, cuando se consolide la recuperación económica, el cambio (por pequeño que haya sido) en los patrones laborales y de cuidados infantiles de padres y madres se revierte a la situación anterior o si se mantienen las tendencias observadas.

En este sentido y dada la timidez de la evolución de la distribución del trabajo extra-doméstico y los cuidados que se puede deducir de los resultados aquí obtenidos, no dejan de ser necesarias las políticas en el ámbito laboral y educativo que favorezcan una asignación del trabajo en 
el hogar más equitativa, que permita a madres y padres desarrollar sus carreras y disfrutar de la maternidad y paternidad.

\section{Bibliografía}

Aguiar, M. A., Hurst, E. y Karabarbounis, L. (2011), Time use during recessions (No. w17259), National Bureau of Economic Research.

Akerlof, G. A., y Kranton, R.E. (2000), "Economics and identity", The Quarterly Journal of Economics, 115(3), 715-753.

Álvarez, B. y Miles, D. (2003), "Gender effect on housework allocation: Evidence from Spanish two-earner couples", Journal of Population Economics, 16(2), 227-242.

Ajenjo, M. y García-Román, J. (2014), "Cambios en el uso del tiempo de las parejas ¿Estamos en el camino hacia una mayor igualdad?”, Revista Internacional de Sociología, $72(2), 453-476$.

Baizán, P., y González, M. J. (2007), “Las escuelas infantiles son la solución? El efecto de la disponibilidad de escuelas infantiles (0-tres años) en el comportamiento laboral femenino", Situación Social de España, 2, 411-443.

Barbeta-Viñas, M. y Cano, T. (2017), “¿Hacia un nuevo modelo de paternidad? Discursos sobre el proceso de implicación paterna en la España urbana”, Revista Española de Investigaciones Sociológicas, 15(3), 13-30.

Becker, G.S. (1981), A treatise on the family, Harvard University Press, Cambridge, MA.

Berik, G. y Kongar, E. (2013), "Time allocation of married mothers and fathers in hard times: The 2007-09 US recession", Feminist Economics, 19(3), 208-237.

Bittman, M., England, P., Folbre, N., Sayer, L. y Matheson, G. (2003), "When does gender trump money? Bargaining and time household work", American Journal of Sociology, 109, 186-214.

Borra, C. (2010), "Childcare costs and Spanish mothers' labour force participation", Hacienda Pública Española/Revista de Economía Pública, 194(3), 9-40.

Borra, C. y Palma, L. (2009), "Child care choices in Spain", Journal of Family and Economic Issues, 30(4), 323.

Chaudry, A., Henly, J. y Meyers, M. (2010), "Conceptual frameworks for child care decision-making. White Paper", Administration for Children \& Families.

Chiuri, M. (2000), "Quality and demand of child care and female labour supply in Italy", Labour, 14(1), 97-118.

Coltrane, S. (2000), "Research on household labor: Modeling and measuring the social embeddedness of Routine Family Work", Journal of Marriage and the Family, 6, 12081233 .

(C) Revista de Economía Laboral 
Connelly, R. (1992), "The effect of child care costs on married women's labor force participation", The Review of Economics and Statistics, 74(1), 83-90.

Del Boca, D. y Vuri, D. (2007), "The mismatch between employment and child care in Italy: the impact of rationing", Journal of Population Economics, 20(4), 805-832.

García-Mainar, I., Molina, J.A. y Montuenga, V.M. (2011), "Gender differences in childcare: time allocation in five European countries", Feminist Economics, 17(1), 119150 .

García-Morán, E. y Kuehn, Z. (2012), "Relaciones entre generaciones y mercado de trabajo. La importancia de los abuelos en la participación laboral de las madres", Panorama Social, 15, 79-92.

García-Román, J. y Cortina, C. (2016), "Family time of couples with children: Shortening gender differences in parenting?", Review of Economics of the Household, 14(4), 921-940.

Gimenez-Nadal, J.I. y Molina, J.A. (2013), "Parents' education as a determinant of educational childcare time", Journal of Population Economics, 26(2), 719-749.

González, M.J. y Jurado-Guerrero, T. (2009), “Cuándo se implican los hombres en las tareas domésticas?: Un análisis de la Encuesta de Empleo del Tiempo”, Panorama Social, $10,65-81$.

Gracia, P. (2014), "Fathers' child care involvement and children's age in Spain: A time use study on differences by education and mothers' employment", European Sociological Review, 30(2), 137-150.

Gracia, P. y Esping-Andersen, G. (2015), "Fathers' child care time and mothers' paid work: A cross-national study of Denmark, Spain, and the United Kingdom", Family Science, 6(1), 270-281.

Gracia, P. y Kalmijn, M. (2016), "Parents' family time and work schedules: The split-shift schedule in Spain”, Journal of Marriage and Family, 78(2), 401-415.

Grossbard, S. (2015), The marriage motive: A price theory of marriage, Springer New York.

Gutiérrez-Domènech, M. (2010), "Parental employment and time with children in Spain", Review of Economics of the Household, 8(3), 371-391.

Izquierdo, M. y Regil, A. (2017), "La evolución del empleo y del paro en 2016, según la Encuesta de Población Activa”, Banco de España - Notas Económicas (31 de enero de 2017).

Legazpe, N. y Davia, M.A. (2015), "Female employment and child care in Spain through the Great Recession”, XI Jornadas de Economía Laboral, Universitat Autònoma de Barcelona.

Llorent, V. (2013), "La educación infantil en Alemania, España, Francia e Inglaterra. estudio comparado”, Revista Española De Educación Comparada, 21, 29-58.

Mamolo, M., Coppola, L. y Di Cesare, M. (2011), "Formal childcare use and household socio-economic profile in France, Italy, Spain and UK", Population Review, 50(1), 170194. 
Meil, G. y Rogero-García, J. (2015), "Does paternal childcare replace grandparental support in dual-earner families?", Family Science, 6(1), 31-37.

Morrill, M.S. y Pabilonia, S.W. (2015), "What effects do macroeconomic conditions have on the time couples with children spend together?", Review of Economics of the Household, 13(4), 791-814.

Morrissey, T.W. (2017), "Child Care and parent labor force participation: a review of the research literature", Review of Economics of the Household, 15(1), 1-24.

Pailhe, A. y Solaz, A. (2008), "Time with children: do fathers and mothers replace each other when one parent is unemployed?", European Journal of Population, 24:, 211-36.

Powell, L.M. (2002), "Joint labor supply and childcare choice decisions of married mothers", The Journal of Human Resources, 37(1), 106-128.

Ribar, D.C. (1995), "A structural model of child care and the labor supply of married women”, Journal of Labor Economics, 13(3), 558-597.

Rodríguez, A. y Ugidos, A. (2017), “Tiempos, trabajos y desigualdad de género en la Comunidad Autónoma del País Vasco y el Estado español”, Ekonomiaz, 91(1), 164-207.

Roodman, D.M. (2011), "Fitting fully observed recursive mixed-process models with cmp", Stata Journal, 11(2), 159-206.

Sevilla-Sanz, A., Gimenez-Nadal, J.I. y Fernández, C. (2010), "Gender roles and the division of unpaid work in Spanish households", Feminist Economics, 16(4), 137-184.

Suárez, M. J. (2013), "Working mothers' decisions on childcare: The case of Spain”, Review of Economics of the Household, 11(4), 545-561.

Tekin, E. (2007), "Childcare subsidies, wages, and employment of single mothers", Journal of Human Resources, 42(2), 509-549.

Van der Lippe, T., Treas, J. y Norbutas, L. (2017), "Unemployment and the division of housework in Europe", Work, Employment and Society, (on line frst, DOI: 10.1177/0950017017690495).

Van Gameren, E. y Ooms, I. (2009), "Childcare and labor force participation in the Netherlands: The Importance of attitudes and opinions", Review of the Economics of the Household, 7(4), 395-421.

Wooldridge, J.M. (2010), Econometric analysis of cross section and panel data, Second Edition, MIT Press.

(C) Revista de Economía Laboral 
Tabla 2: Modelo Tobit multivariado (efectos marginales).

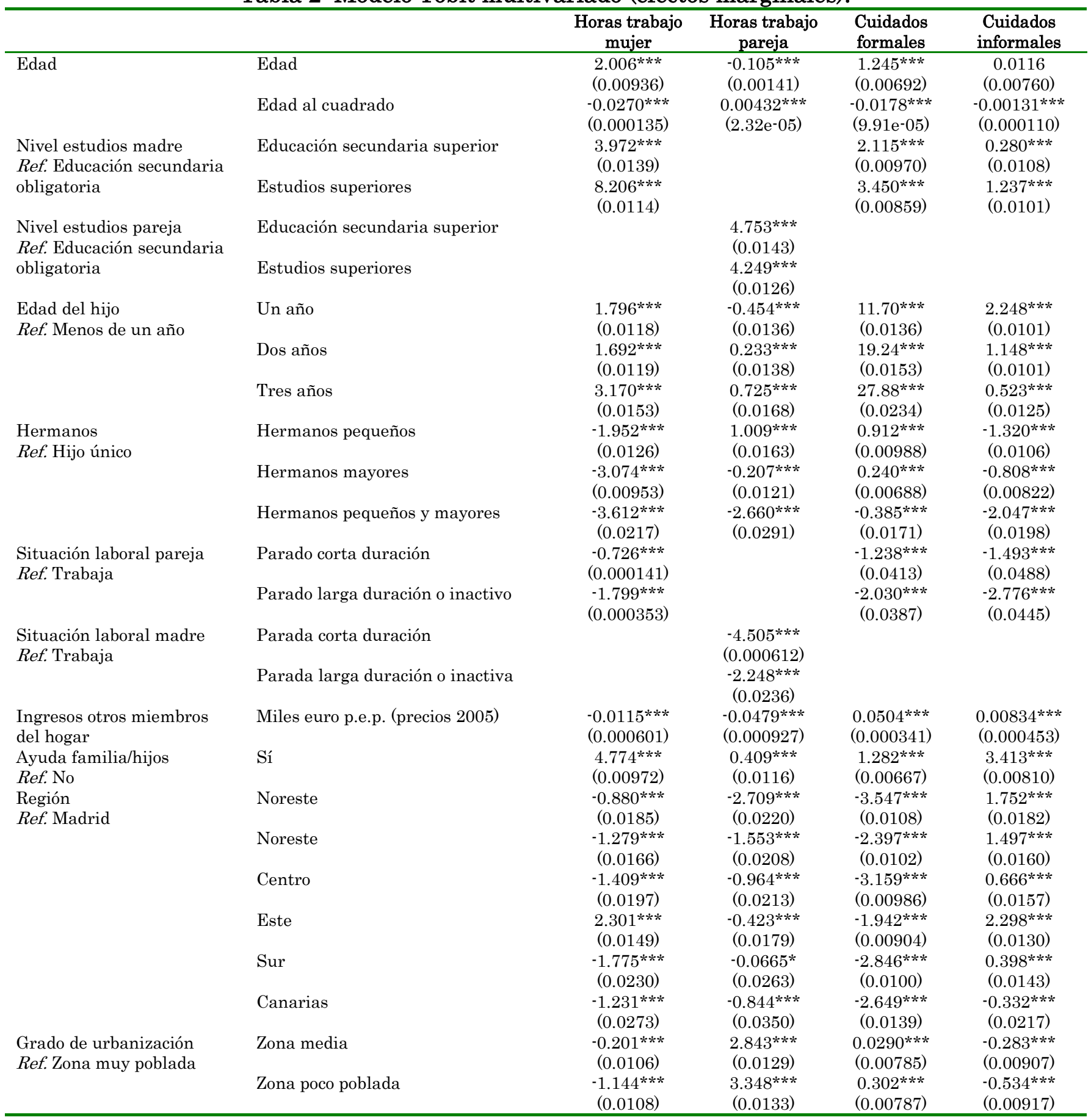


Tabla 2: Modelo Tobit multivariado (efectos marginales) (continuación).

\begin{tabular}{|c|c|c|c|c|c|}
\hline & & $\begin{array}{c}\text { Horas trabajo } \\
\text { mujer }\end{array}$ & $\begin{array}{c}\text { Horas trabajo } \\
\text { pareja }\end{array}$ & $\begin{array}{l}\text { Cuidados } \\
\text { formales }\end{array}$ & $\begin{array}{c}\text { Cuidados } \\
\text { informales }\end{array}$ \\
\hline \multirow[t]{2}{*}{ Tasa de paro } & Tasa de paro femenino & $\begin{array}{l}-0.182^{* * * *} \\
(0.00147)\end{array}$ & & & \\
\hline & Tasa de paro masculino & & $\begin{array}{l}-0.464^{* * * *} \\
(0.00221)\end{array}$ & & \\
\hline \multirow{10}{*}{$\begin{array}{l}\text { Año } \\
\text { Ref. Año } 2005\end{array}$} & Año 2006 & $0.579 * * *$ & $-1.915^{* * * *}$ & $0.728^{* * * *}$ & $0.167^{* * *}$ \\
\hline & Año 2007 & $\begin{array}{c}(0.0200) \\
-0.356^{* * *} \\
(0.0195)\end{array}$ & $\begin{array}{c}(0.0223) \\
-1.934^{* * *} \\
(0.0223)\end{array}$ & $\begin{array}{c}(0.0143) \\
0.730^{* * *} \\
(0.0144)\end{array}$ & $\begin{array}{c}(0.0153) \\
-0.403^{* * *} \\
(0.0150)\end{array}$ \\
\hline & Año 2008 & $\begin{array}{l}2.643^{* * * *} \\
(0.0211)\end{array}$ & $\begin{array}{c}-1.201^{* * * *} \\
(0.0236)\end{array}$ & $\begin{array}{l}1.027^{* * *} \\
(0.0146)\end{array}$ & $\begin{array}{c}-0.837^{* * * *} \\
(0.0148)\end{array}$ \\
\hline & Año 2009 & $\begin{array}{l}2.830 * * * \\
(0.0235)\end{array}$ & $\begin{array}{c}-0.799 * * * \\
(0.0326)\end{array}$ & $\begin{array}{c}0.990 * * * \\
(0.0156)\end{array}$ & $\begin{array}{c}-0.885^{* * *} \\
(0.0158)\end{array}$ \\
\hline & Año 2010 & $\begin{array}{l}4.336 * * * \\
(0.0263)\end{array}$ & $\begin{array}{l}1.486 * * * \\
(0.0363)\end{array}$ & $\begin{array}{l}1.716^{* * *} \\
(0.0158)\end{array}$ & $\begin{array}{c}-0.870 * * * \\
(0.0153)\end{array}$ \\
\hline & Año 2011 & $\begin{array}{l}4.388^{* * *} \\
(0.0276)\end{array}$ & $\begin{array}{c}-0.402^{* * * *} \\
(0.0378)\end{array}$ & $\begin{array}{l}1.380 * * * \\
(0.0160)\end{array}$ & $\begin{array}{c}-1.786^{* * *} \\
(0.0154)\end{array}$ \\
\hline & Año 2012 & $\begin{array}{l}4.716^{* * * *} \\
(0.0313)\end{array}$ & $\begin{array}{c}-1.252^{* * * *} \\
(0.0442)\end{array}$ & $\begin{array}{c}-1.306^{* * *} \\
(0.0146)\end{array}$ & $\begin{array}{c}-2.184^{* * *} \\
(0.0165)\end{array}$ \\
\hline & Año 2013 & $\begin{array}{l}4.817 * * * \\
(0.0331)\end{array}$ & $\begin{array}{c}-0.338 * * * \\
(0.0458)\end{array}$ & $\begin{array}{c}0.702 * * * \\
(0.0162)\end{array}$ & $\begin{array}{c}-3.169^{* * * *} \\
(0.0157)\end{array}$ \\
\hline & Año 2014 & $\begin{array}{l}4.705^{* * *} \\
(0.0315)\end{array}$ & $\begin{array}{c}-1.838 * * * \\
(0.0413)\end{array}$ & $\begin{array}{l}1.258^{* * *} \\
(0.0171)\end{array}$ & $\begin{array}{c}-1.006^{* * *} \\
(0.0175)\end{array}$ \\
\hline & Año 2015 & $\begin{array}{l}5.914^{* * *} \\
(0.0307)\end{array}$ & $\begin{array}{l}1.128^{* * *} \\
(0.0377)\end{array}$ & $\begin{array}{c}-0.926^{* * *} \\
(0.0133)\end{array}$ & $\begin{array}{c}-2.255^{* * *} \\
(0.0148)\end{array}$ \\
\hline Coeficientes de & $\rho 21$ (sign.) & \multicolumn{4}{|c|}{$0.0972 * * *(0.00100)$} \\
\hline correlación entre los & $\rho 31$ (sign.) & \multicolumn{4}{|c|}{$0.242^{* * *}(0.000581)$} \\
\hline términos de error de & $\rho 41$ (sign.) & \multicolumn{4}{|c|}{$0.408^{* * *}(0.000666)$} \\
\hline las ecuaciones. & $\rho 32$ (sign.) & \multicolumn{4}{|c|}{$0.0357 * * * *(0.00204)$} \\
\hline & $\rho 42$ (sign.) & \multicolumn{4}{|c|}{$0.0903^{* * *}(0.00230)$} \\
\hline & $\rho 34$ (sign.) & \multicolumn{4}{|c|}{$-0.0904 * * *(0.000557)$} \\
\hline Número observaciones & & \multicolumn{4}{|c|}{7.924} \\
\hline
\end{tabular}

Fuente: Encuesta de Condiciones de Vida. Ficheros transversales 2005-2015. Instituto Nacional de Estadística.

(C) Revista de Economía Laboral 


\section{Apéndice}

Tabla A.1: Valores medios de las variables independientes en el modelo multivariante.

\begin{tabular}{|c|c|c|c|c|}
\hline & & Total & $\begin{array}{c}\text { Periodo } \\
\text { crecimiento } \\
(2005-2008 ; 2015)\end{array}$ & $\begin{array}{l}\text { Periodo crisis } \\
(2009-2014)\end{array}$ \\
\hline \multirow[t]{4}{*}{ Horas } & Trabaja mujer & 21,31 & 20,67 & 21,88 \\
\hline & Trabaja pareja & 37,81 & 40,39 & 35,55 \\
\hline & Cuidados formales & 11,55 & 11,19 & 11,88 \\
\hline & Cuidados informales & 5,05 & 5,48 & 4,67 \\
\hline Edad de la mujer & Edad & 34,60 & 34,43 & 34,74 \\
\hline \multirow[t]{3}{*}{$\begin{array}{l}\text { Nivel educativo de la } \\
\text { mujer }\end{array}$} & $\begin{array}{l}\text { Educación secundaria } \\
\text { obligatoria }\end{array}$ & 26,62 & 29,07 & 24,47 \\
\hline & Educación secundaria superior & 22,15 & 22,56 & 21,79 \\
\hline & Estudios superiores & 51,23 & 48,37 & 53,74 \\
\hline Edad de la pareja & Edad & 36,77 & 36,60 & 36,91 \\
\hline \multirow[t]{3}{*}{$\begin{array}{l}\text { Nivel educativo de la } \\
\text { pareja }\end{array}$} & $\begin{array}{l}\text { Educación secundaria } \\
\text { obligatoria }\end{array}$ & 36,40 & 35,92 & 36,82 \\
\hline & Educación secundaria superior & 21,79 & 24,05 & 19,81 \\
\hline & Estudios superiores & 41,81 & 40,03 & 43,37 \\
\hline \multirow[t]{4}{*}{ Edad del hijo } & Menos de un año & 24,69 & 24,97 & 24,44 \\
\hline & Un año & 29,57 & 29,49 & 29,63 \\
\hline & Dos años & 29,91 & 29,48 & 30,29 \\
\hline & Tres años & 15,83 & 16,05 & 15,64 \\
\hline \multirow[t]{4}{*}{ Hermanos } & Hijo único & 46,18 & 46,41 & 45,99 \\
\hline & Hermanos pequeños & 12,26 & 11,79 & 12,67 \\
\hline & Hermanos mayores & 38,51 & 38,25 & 38,75 \\
\hline & Hermanos pequeños y mayores & 3,04 & 3,55 & 2,59 \\
\hline Situación laboral de & Trabaja & 61,64 & 59,75 & 63,30 \\
\hline \multirow[t]{2}{*}{ la mujer } & Parada corta duración & 6,52 & 6,16 & 6,84 \\
\hline & Parada larga duración o inactiva & 31,84 & 34,09 & 29,86 \\
\hline \multirow{3}{*}{$\begin{array}{l}\text { Situación laboral } \\
\text { pareja }\end{array}$} & Trabaja & 88,22 & 93,26 & 83,80 \\
\hline & Parado corta duración & 5,27 & 3,29 & 7,00 \\
\hline & Parado larga duración o inactivo & 6,51 & 3,45 & 9,20 \\
\hline \multirow{4}{*}{$\begin{array}{l}\text { Ingresos otros } \\
\text { miembros del hogar } \\
\text { Ayuda familia/hijos }\end{array}$} & Miles euro p.e.p. (precios 2005) & 13,51 & 13,40 & 13,61 \\
\hline & Miles euro p.e.p. (precios 2005) & 10,21 & 9,54 & 10,80 \\
\hline & No & 67,87 & 68,57 & 67,25 \\
\hline & Sí & 32,13 & 31,43 & 32,75 \\
\hline \multirow[t]{7}{*}{ Región } & Madrid & 13,60 & 13,53 & 13,66 \\
\hline & Noroeste & 8,25 & 8,50 & 8,03 \\
\hline & Noreste & 9,65 & 9,17 & 10,07 \\
\hline & Centro & 12,33 & 12,25 & 12,41 \\
\hline & Este & 28,71 & 28,90 & 28,55 \\
\hline & Sur & 23,20 & 23,36 & 23,05 \\
\hline & Canarias & 4,26 & 4,30 & 4,22 \\
\hline \multirow{3}{*}{$\begin{array}{l}\text { Grado de } \\
\text { urbanización }\end{array}$} & Zona muy poblada & 49,00 & 48,39 & 49,54 \\
\hline & Zona media & 24,14 & 24,13 & 24,14 \\
\hline & Zona poco poblada & 26,86 & 27,48 & 26,31 \\
\hline
\end{tabular}




\begin{tabular}{|c|c|c|c|c|}
\hline \multirow[t]{2}{*}{ Tasa de paro } & Tasa de paro femenino & 17,98 & 13,42 & 21,98 \\
\hline & Tasa de paro masculino & 15,14 & 9,05 & 20,48 \\
\hline \multirow[t]{11}{*}{ Año } & Año 2005 & 9,21 & 19,71 & - \\
\hline & Año 2006 & 9,33 & 19,96 & - \\
\hline & Año 2007 & 9,37 & 20,05 & - \\
\hline & Año 2008 & 9,41 & 20,15 & - \\
\hline & Año 2009 & 8,80 & - & 16,51 \\
\hline & Año 2010 & 8,75 & - & 16,43 \\
\hline & Año 2011 & 8,88 & - & 16,67 \\
\hline & Año 2012 & 8,59 & - & 16,12 \\
\hline & Año 2013 & 8,93 & - & 16,76 \\
\hline & Año 2014 & 9,33 & - & 17,52 \\
\hline & Año 2015 & 9,41 & 20,13 & - \\
\hline \multicolumn{2}{|c|}{ Número de observaciones } & 7.924 & 4.003 & 3.921 \\
\hline
\end{tabular}

Fuente: Encuesta de Condiciones de Vida. Ficheros transversales 2005-2015. Instituto Nacional de Estadística.

(C) Revista de Economía Laboral 


\section{Tabla A.2: Modelo Tobit multivariado en sub-periodos (efectos marginales) (selección).}

\begin{tabular}{|c|c|c|c|c|c|c|c|c|c|}
\hline & \multicolumn{4}{|c|}{$\begin{array}{l}\text { Periodo crecimiento } \\
(2005-2008 \text { y } 2015)\end{array}$} & \multicolumn{4}{|c|}{$\begin{array}{c}\text { Periodo crisis } \\
\text { (2009-2014) }\end{array}$} \\
\hline & & $\begin{array}{c}\text { Horas L } \\
\text { mujer }\end{array}$ & $\begin{array}{c}\text { Horas L } \\
\text { pareja }\end{array}$ & $\begin{array}{l}\text { Cuidados } \\
\text { formales }\end{array}$ & $\begin{array}{c}\text { Cuidados } \\
\text { informales }\end{array}$ & $\begin{array}{c}\text { Horas L } \\
\text { mujer }\end{array}$ & $\begin{array}{c}\text { Horas L } \\
\text { pareja }\end{array}$ & $\begin{array}{l}\text { Cuidados } \\
\text { formales }\end{array}$ & $\begin{array}{l}\text { Cuidados } \\
\text { informales }\end{array}$ \\
\hline \multirow{4}{*}{$\begin{array}{l}\text { Situación } \\
\text { laboral } \\
\text { pareja } \\
\text { Ref. } \\
\text { Trabaja }\end{array}$} & Parada & $-0.562^{* * *}$ & & $-1.217^{* * *}$ & $-1.465^{* * *}$ & $-0.0762^{* * *}$ & & $-1.062^{* * *}$ & $-1.372^{* * *}$ \\
\hline & $\begin{array}{l}\text { corta } \\
\text { duración }\end{array}$ & $(0.0169)$ & & $(0.0328)$ & $(0.0537)$ & $\left(1.96 \mathrm{e}^{-05)}\right.$ & & $(0.0392)$ & $(0.000542)$ \\
\hline & Parada & $-3.709 * * *$ & & $-0.521^{* * *}$ & $-2.048^{* * *}$ & $-0.253^{* * *}$ & & $-2.203^{* * *}$ & $-3.288^{* * *}$ \\
\hline & $\begin{array}{l}\text { larga } \\
\text { duración / } \\
\text { inact. }\end{array}$ & (0.107) & & $(0.0494)$ & $(0.0670)$ & $\left(6.53 \mathrm{e}^{-05}\right)$ & & $(0.0354)$ & $(0.0253)$ \\
\hline \multirow{5}{*}{$\begin{array}{l}\text { Situación } \\
\text { laboral } \\
\text { madre } \\
\text { Ref. } \\
\text { Trabaja }\end{array}$} & Parada & & - & & & & & & \\
\hline & corta & & $4.182^{* * * *}$ & & & & $-4.048^{* * *}$ & & \\
\hline & duración & & $(0.146)$ & & & & (0.000978) & & \\
\hline & Parada & & & & & & & & \\
\hline & $\begin{array}{l}\text { larga } \\
\text { duración / } \\
\text { inact. }\end{array}$ & & $\begin{array}{c}1.911^{* * * *} \\
(0.127)\end{array}$ & & & & $\begin{array}{c}-1.675^{* * *} \\
(0.0376)\end{array}$ & & \\
\hline \multirow{6}{*}{$\begin{array}{l}\text { Coeficient } \\
\text { es de } \\
\text { correlació } \\
\mathrm{n} \text { entre } \\
\text { los } \\
\text { términos } \\
\text { de error }\end{array}$} & $\rho 21$ (sign.) & \multicolumn{2}{|c|}{$-0.0635^{* * *}$} & \multicolumn{2}{|c|}{$(0.00689)$} & \multicolumn{2}{|c|}{$0.162^{* * *}$} & \multicolumn{2}{|c|}{$(0.00156)$} \\
\hline & $\rho 31$ (sign.) & \multicolumn{2}{|c|}{$0.278^{* * *}$} & \multirow{2}{*}{\multicolumn{2}{|c|}{$(0.00745)$}} & \multicolumn{2}{|c|}{$0.254^{* * * *}$} & \multicolumn{2}{|c|}{$(0.000840)$} \\
\hline & $\rho 41$ (sign.) & \multicolumn{2}{|c|}{$0.440 * * *$} & & & \multicolumn{2}{|c|}{$0.433^{* * *}$} & \multicolumn{2}{|c|}{$(0.000861)$} \\
\hline & $\rho 32$ (sign.) & \multicolumn{2}{|c|}{$0.0188 * * *$} & \multicolumn{2}{|c|}{$\begin{array}{c}(0.0118) \\
(0.00174)\end{array}$} & \multicolumn{2}{|c|}{$0.0565 * * *$} & \multicolumn{2}{|c|}{$(0.00210)$} \\
\hline & $\rho 42$ (sign.) & \multicolumn{2}{|c|}{-0.000183} & & \multicolumn{2}{|c|}{$0.0972^{* * *}$} & \multicolumn{2}{|c|}{$(0.00162)$} \\
\hline & $\rho 34$ (sign.) & \multicolumn{2}{|c|}{$-0.226^{* * *}$} & \multicolumn{2}{|c|}{$\begin{array}{l}(0.00402) \\
(0.00644)\end{array}$} & \multicolumn{2}{|c|}{$-0.0635^{* * *}$} & \multicolumn{2}{|c|}{$(0.000792)$} \\
\hline \multicolumn{2}{|c|}{$\begin{array}{l}\text { Número de } \\
\text { observaciones }\end{array}$} & \multicolumn{4}{|c|}{4.003} & \multicolumn{4}{|c|}{3.921} \\
\hline
\end{tabular}

Fuente: Encuesta de Condiciones de Vida. Ficheros transversales 2005-2015. Instituto Nacional de Estadística. Nota: estos modelos tienen la misma especificación que la mostrada en la Tabla 2. El resto de efectos marginales, correspondientes a las especificaciones descritas en la Tabla 2, no se muestran aquí por razones de espacio. 